\title{
Acute Complicated Type B Aortic Dissection during the New York City COVID-19 Surge.
}

\author{
Shinichiro Ikeda ${ }^{1}$, Michael Shih ${ }^{1}$, Robert Rhee ${ }^{1}$, and Benjamin Youdelman ${ }^{1}$ \\ ${ }^{1}$ Maimonides Medical Center
}

May 8, 2020

\begin{abstract}
The impact of the COVID-19 pandemic in New York City (NYC) is dramatic. COVID-19 cases surged, hospitals expanded to meet capacity, and NYC remains the global epicenter of this pandemic. During this unprecedented time, a young woman with known Marfan syndrome presented with an acute complicated type B aortic dissection to our Aortic Center. Using the Provisional Extension to Induce Complete Attachment technique, we treated this patient and quickly discharged her the next day to decrease the risk of COVID-19 infection. Her progress was monitored using frequent phone calls and one office visit at two weeks.
\end{abstract}

\section{Introduction}

The impact of the COVID-19 pandemic in New York City is dramatic. COVID-19 cases surged and hospitals expanded to meet the need. Elective surgical cases were cancelled due to concern about hospital beds and the risk of perioperative COVID-19 infection. This same concern contributed to the dramatic decrease in non-COVID-19 emergency cases presenting to the hospital which was associated with a dramatic increase in the at-home deaths during this same period according to the contact with [Reference is contact with FDNY regarding EMS at home death findings March-April 2020]. During this unprecedented time, a young woman with known Marfan Syndrome (MFS) presented with an acute complicated type B aortic dissection (ACTBAD).

Thoracic endovascular aortic repair (TEVAR) is the procedure of choice for ACTBAD. TEVAR is not recommended for Marfan syndrome (MFS) patients, but it could be potentially beneficial under critical circumstances $[1,2]$. Use of the Provisional Extension to Induce Complete Attachment (PETTICOAT) technique has been demonstrated to decrease the risk of spinal cord ischemia in comparison to use of covered stent grafts in similar settings [3-6].

\section{Case presentation}

A 35-year-old female with MFS presented with chest pain, abdominal pain, and numbness in right extremity. She was hemodynamically stable. On examination, her right leg was colder than the left one with no dopplerable distal pulses. Computed tomography (CT) scan showed Stanford type B aortic dissection (TBAD) from proximal descending aorta to the right common iliac artery (CIA), which was occluded. The true lumen (TL) was 95\% compressed by false lumen (FL) (Fig 1A and B). The celiac artery (CA), superior mesenteric artery (SMA), and left renal artery (RA) were perfused from FL. The flow to the right renal and CIAs were significantly decreased due to TL compression (Fig 1C and D). She was taken emergently to the operating room (OR). The procedure was performed under general anesthesia. Bilateral common femoral arteries were accessed percutaneously. Intravascular ultrasound examination was performed to confirm true lumen placement of the wires (Fig 2A and B). A $28 \mathrm{~mm}$ x $109 \mathrm{~mm}$ Zenith Alpha Thoracic Endovascular Graft (Cook Medical, Bloomington, Ind) was deployed into the proximal descending aorta covering the primary 
tear site (Fig 2C). Significant compression of TL persisted (Fig 2D). Therefore, a $36 \times 180 \mathrm{~mm}$ bare metal Zenith Dissection Endovascular Stent (Cook Medical, Bloomington, Ind) was placed down to the CA (Fig 2E). An aortogram confirmed antegrade filling of the CA, SMA, and right RA. The left RA was supplied from FL via distal fenestration (Fig 2 F). The previously collapsed right CIA was fully re-expanded and the right pedal pulses became palpable. The patient was extubated and transferred to intensive care unit. The patient was stable overnight with no neurological deficits and normal creatinine level. Due to high risk of infection with COVID-19, she was discharged home 20 hours after surgery. Follow up was performed via frequent phone calls and she was seen in the office at two weeks.

\section{Comments:}

The rate of spinal cord ischemia after TEVAR is 6-10\% [3, 5]. In contrast, in the Study of Thoracic Aortic Type B Dissection Using Endoluminal Repair (STABLE I), favorable results were found with the Zenith Dissection Endovascular System (Cook Medical, Bloomington, Ind) with a 30-day paraplegia rate of $1.8 \%$ [4]. Less disruption of spinal cord perfusion with the use of bare metal stent, continued false lumen compression to encourage thrombosis and remodeling [7].

There is limited data on TEVAR/EVAR for MFS patients and only a few case reports presented PETTICOAT technique for MFS patients [6]. A study reported that the rate of the primary treatment failure in MFS patients treated with TEVAR/EVAR was $44 \%$ due to endoleaks [2]. We present a patient who is a member of a subset of MFS patients who can benefit from TEVAR as a rescue therapy. This was true both because of the clinical presentation, and the timing of her presentation during the COVID-19 pandemic surge. If there was a problem that made her unable to be extubated, we would have to stay in the OR on the anesthesia machine until a ventilator was delivered as there was no available ventilator in the hospital. The patient will be followed closely for the need for surgical repair.

\section{Conclusion}

Rapid TEVAR with the PETTICOAT technique was effective to expand the TL, reperfuse the visceral organs and lower extremities, prevent spinal cord ischemia, and allowed prompt discharge during the COVID-19 pandemic. Standard postoperative protocols were abandoned effectively for patient safety.

\section{References}

1. Svensson LG, Kouchoukos NT, Miller DC, et al. Expert consensus document on the treatment of descending thoracic aortic disease using endovascular stent grafts. Ann Thorac Surg 2008; 85:S1-41

2. Waterman AL, Feezor RJ, Lee WA, et al. Endovascular treatment of acute and chronic aortic pathology in patients with Marfan syndrome. J Vasc Surg 2012; 55:1234-41.

3. Feezor RJ, Martin TD, Hess PJ, et al. Extent of aortic coverage and incidence of spinal cord ischemia after thoracic endovascular aneurysm repair. Ann Thorac Surg. 2008; 86:1809-1814.

4. Lombardi JV, Cambria RP, Nienaber CA, et al. Five-year results from the study of Thoracic Aortic Type B Dissection Using Endoluminal Repair (STABLE I) study of endovascular treatment of complicated type B aortic dissection using a composite device design. J Vasc Surg. 2019; 70:1072-81.

5. White RA, Miller DC, Criado FJ, et al. Report on the results of thoracic endovascular aortic repair for acute complicated type B aortic dissection at 30 days and 1 year from a multidisciplinary subcommittee of the Society for Vascular Surgery Outcomes Committee. J Vasc Surg. 2011; 53:1082-90.

6. Antonello M, Squizzato F, Colacchio C, Taqlialavoro J, Grego F, Piazza M. The PETTICOAT technique for complicated acute stanford type B aortic dissection using a tapered self-expanding nitinol device as distal uncovered stent. Ann Vasc Surg 2017; 42:308-316.

7. Leshnower BG, Duwayri YM, Chen EP, et al. Aortic remodeling after endovascular repair of complicated acute type B aortic dissection. Ann Thorac Surg. 2017; 103:1878-1885. 


\section{Declaration of conflicting interests:}

The authors declare that there is no conflict of interest.

\section{Figure legends:}

Fig. 1 Preoperative computed tomography scan which shows (A) dissection started at the proximal descending aorta. The TL was significantly compressed by the FL. (B) The entry tear was seen in the mid-descending aorta. (C) Right kidney flow was diminished as the TL is significantly compressed. (D) A flow of the Rt. CIA decreased.

TL: True lumen; FL: False lumen; Rt. CIA: Right common iliac artery Fig 2. Intraoperative angiogram demonstrates (A) angiography from the TL. The flow of the CA and the SMA were decreased. (B) The flow in the Rt. RA and the Rt. CIA decreased. (C) A 28 x 109 mm Zenith Alpha Thoracic Endovascular Graft (Cook Medical, Bloomington, Ind) was deployed into the proximal descending aorta covering the primary tear site (Between the red lines). (D) The TL was still compressed from the FL through the visceral arteries segment and infrarenal segment with improved visceral perfusion. (E) After TEVAR and the distal bare metal stent (Between the red lines), the flow of CA and SMA improved. The flow into the FL in the descending aorta disappeared. (F) The flow of Rt. RA and Rt. CIA was clearly seen and the left RA was perfused from the FL. TL: True lumen, FL: False lumen, Rt. RA: Right renal artery, Rt. CIA: Right common iliac artery, CA: Celiac artery, TEAR: Thoracic endovascular aortic repair

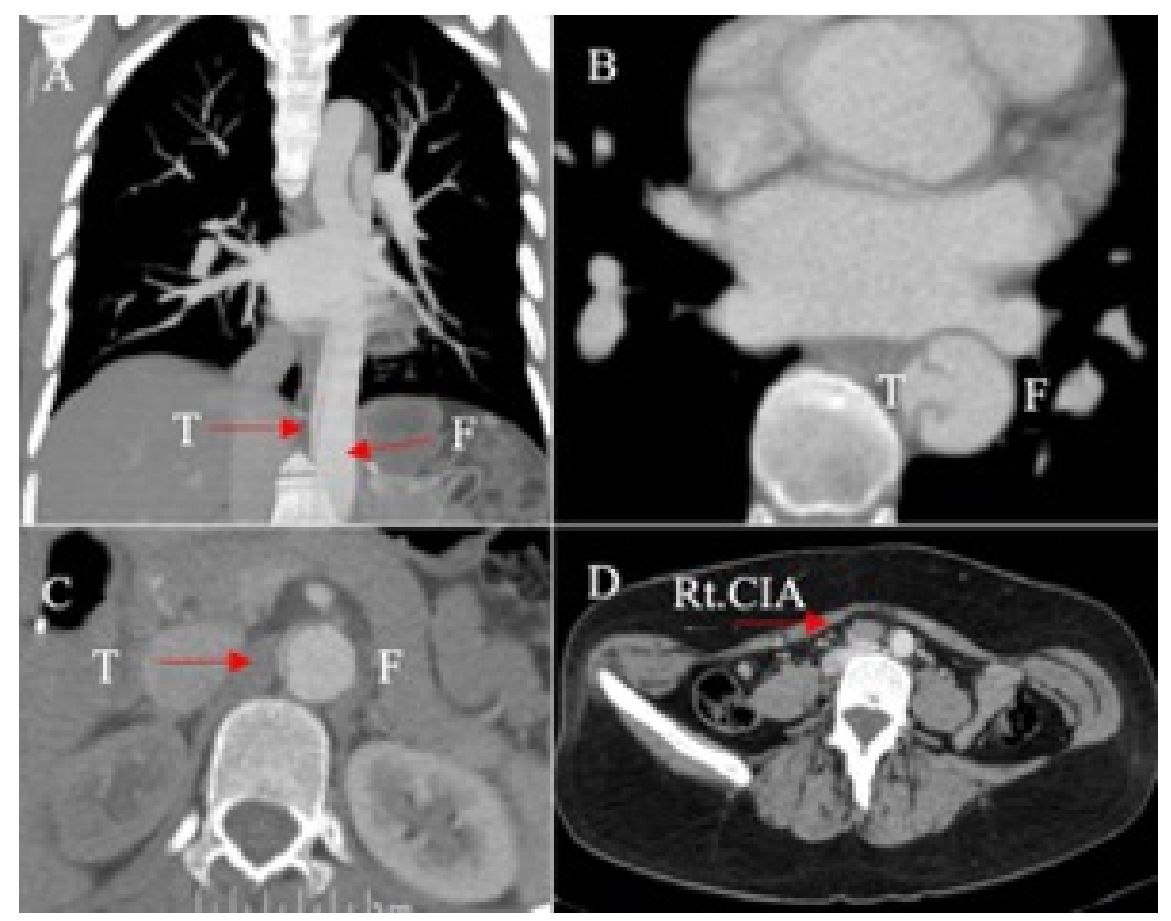




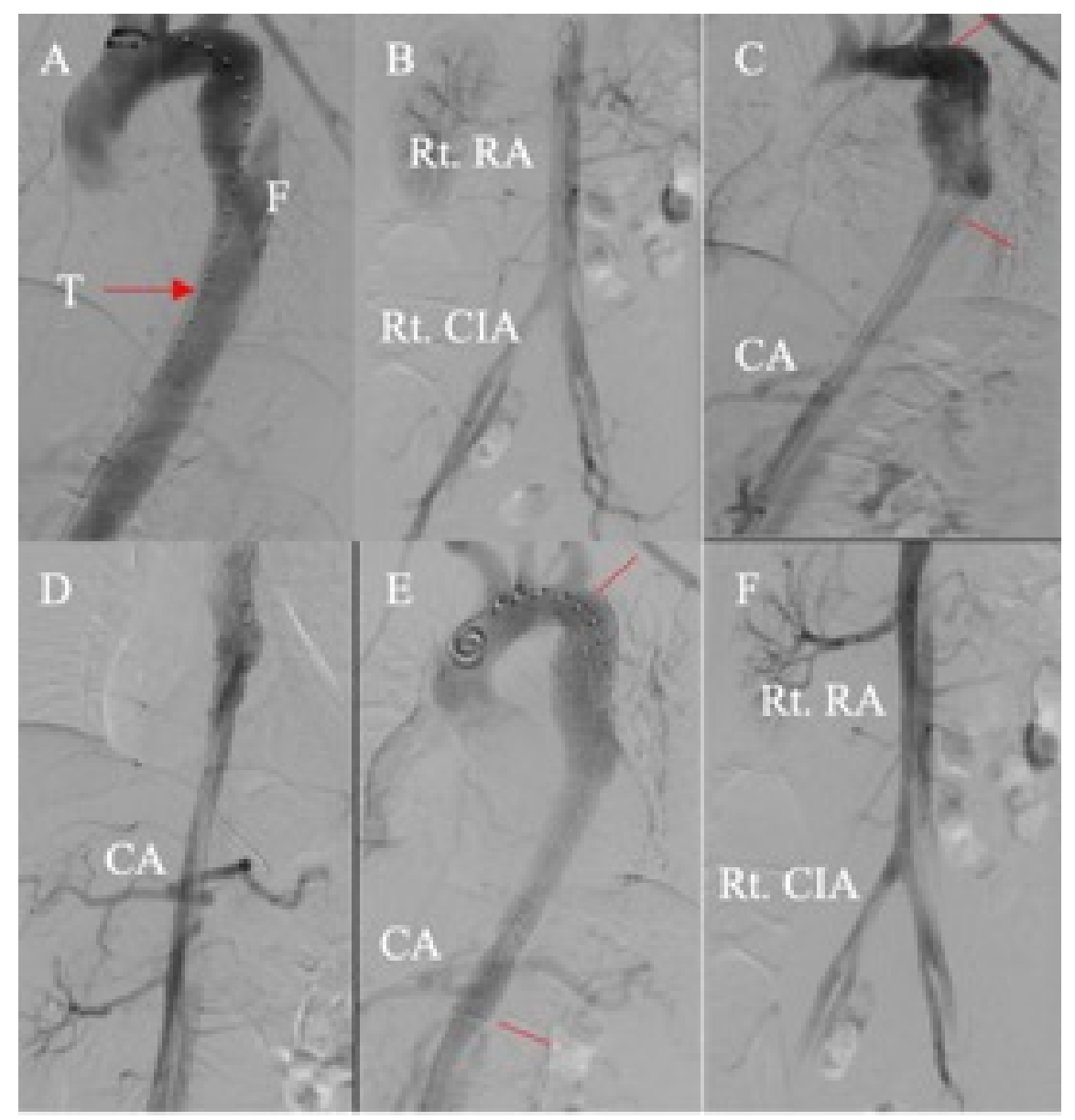

